\title{
NÚMEROS COMPLEXOS E QUATÉRNIOS: DOS CONTEXTOS HISTÓRICOS AO USO DO SOFTWARE EDUCATIVO LIVRE GEOGEBRA E SOFTWARE LIVRE ON-LINE QUATERNION
}

\author{
COMPLEX NUMBERS AND QUATERNIONS: FROM HISTORICAL \\ CONTEXTS TO THE USE OF GEOGEBRA FREE EDUCATIONAL \\ SOFTWARE AND ON-LINE FREE SOFTWARE QUATERNION
}

\author{
Alessandro Ribeiro da Silva ${ }^{1}$ \\ Renato César da Silva² \\ Edivaldo Romanini ${ }^{3}$
}

\begin{abstract}
RESUMO: O presente artigo relata um trabalho desenvolvido no âmbito do Programa de Educação Tutorial (PET Conexões de Saberes Matemática), do Curso de Licenciatura em Matemática da Universidade Federal de Mato Grosso do Sul (UFMS) - Campus de Três Lagoas (CPTL). O objetivo é apresentar de forma breve o contexto histórico da formulação dos Números Complexos e dos Quatérnios, bem como a utilização dos Softwares Educativos Livre GeoGebra e QUATERNION como recursos pedagógicos, facilitando a compreensão das definições, propriedades e conceitos relacionados a esses conjuntos. O trabalho apresenta também resultados de uma pesquisa feita com estudantes do curso de Licenciatura em Matemática sobre o uso de tecnologia para o ensino de números complexos e quatérnios. Através da pesquisa foi possível perceber a eficiência de usar recursos tecnológicos, em especial os Softwares livres em questão, bem como proporcionar ao estudante pesquisador um novo conhecimento da generalização dos Números Complexos (caracterizados pelos Quatérnios).
\end{abstract}

PALAVRAS-CHAVE: Números Complexos. Softwares Educativos Livres. Ensino de Matemática.

ABSTRACT: The present article reports a work developed within the ambit of the Tutorial Education Program (PET Mathematical Knowledge connections), of the Degree Course in Mathematics at the Federal University of Mato Grosso do Sul (UFMS) - Três Lagoas Campus (CPTL). The objective is to briefly present the historical context of the formulation of Complex Numbers and Quaternions, as well as the use of the Free Educational Software GeoGebra and QUATERNION as pedagogical resources, facilitating the understanding of definitions, properties and concepts related to these sets. The paper also presents results from a research done with undergraduate mathematics students on the use of technology for teaching complex numbers and quaternions. Through the research it was possible to realize the efficiency of using technological resources, in particular the free Software in question, as well as providing the student researcher with new knowledge of the generalization of Complex Numbers (characterized by the Quaternions).

KEYWORDS: Complex Numbers. Free Educational Software. Mathematics Teaching.

\footnotetext{
${ }^{1}$ Universidade Federal de Mato Grosso do Sul, Brasil. E-mail: alessandro.ribeiro@ufms.br.

(iD) https://orcid.org/0000-0001-8012-2072

2 Universidade Federal de Mato Grosso do Sul, Brasil. E-mail: renato.silva@ufms.br

(iD) https://orcid.org/0000-0002-7931-7785

3 Universidade Federal de Mato Grosso do Sul, Brasil. E-mail: edivaldo.romanini@ufms.br

(D) https://orcid.org/0000-0001-5382-0326
}

- Informações completas da obra no final do artigo 
Introdução

A consolidação das operações com os Números Complexos, teve seu início durante o século XVI, quando os matemáticos, Scipione Del Ferro (1465 - 1526) e Niccolò Fontana Tartaglia (1500 - 1557), desenvolveram a fórmula para as soluções de equações cúbicas reduzidas, sem o termo do segundo grau. Porém, segundo contextos históricos é Girolamo Cardano (1501-1576), quem demonstrou pela primeira vez em sua obra conhecida como "Ars Magna" publicado em Nuremberg (1545) na Alemanha, tal método. Cardano é surpreendido ao passo que começa a lidar com "raízes quadradas de números negativos segundo as regras usuais da álgebra de então". Logo, "nessa época nem os números negativos eram compreendidos com muita clareza" (NEVES, 2014, p. 3).

A equação geral do terceiro grau pode ser determinada pela expressão convencionada $a x^{3}+b x^{2}+c x+d=0$, com $a \neq 0$. Com a utilização dessa fórmula é possível encontrar todas as raízes do terceiro grau, porém para que isso seja possível, é necessário converter a equação cúbica completa, numa equação cúbica sem o termo do segundo grau. Dessa forma, a equação cúbica do terceiro grau $a x^{3}+b x^{2}+c x+d=0$, é reduzida para $y^{3}+p y+q=0$ (GUEDES JUNIOR, 2016).

Considerando $y=u+v$, pode-se encontrar a equação do segundo grau, onde $u^{3} \mathrm{e}$ $v^{3}$ são as raízes:

$$
\begin{gathered}
y^{3}+p y+q=0 \\
\left(u^{3}+v^{3}\right)=-q e u^{3} v^{3}=-\frac{p^{3}}{27}
\end{gathered}
$$

Sendo $u^{3}$ e $v^{3}$ as raízes, então:

$$
\begin{aligned}
& u^{3}=\frac{-q}{2}+\sqrt{\frac{b^{2}}{4}+\frac{p^{3}}{27}} \Rightarrow \sqrt[3]{u^{3}}=\sqrt[3]{\frac{-q}{2}+\sqrt{\frac{b^{2}}{4}+\frac{p^{3}}{27}}} \Rightarrow u=\sqrt[3]{\frac{-q}{2}+\sqrt{\frac{b^{2}}{4}+\frac{p^{3}}{27}}} \\
& v^{3}=\frac{-q}{2}-\sqrt{\frac{b^{2}}{4}+\frac{p^{3}}{27}} \Rightarrow \sqrt[3]{v^{3}}=\sqrt[3]{\frac{-q}{2}-\sqrt{\frac{b^{2}}{4}+\frac{p^{3}}{27}}} \Rightarrow v=\sqrt[3]{\frac{-q}{2}-\sqrt{\frac{b^{2}}{4}+\frac{p^{3}}{27}}}
\end{aligned}
$$

Como na equação $y^{3}+p y+q=0$, foi considerado que $y=u+v$. Assim segue:

$$
y=\sqrt[3]{\frac{-q}{2}+\sqrt{\frac{b^{2}}{4}+\frac{p^{3}}{27}}}+\sqrt[3]{\frac{-q}{2}-\sqrt{\frac{b^{2}}{4}+\frac{p^{3}}{27}}} .
$$


A equação anterior ficou conhecida como Fórmula de Cardano, Tartaglia e Del Ferro. Após sua demonstração, conforme já mencionado, Cardano apresentou-se surpreso ao lidar com raízes de números negativos que eram números vistos com desconfiança, não levando adiante os estudos de números negativos, dessa forma, segundo Neves (2014):

\begin{abstract}
Tal tarefa foi levada a cabo por Rafael Bombelli (1526-1572), que dedicou boa parte de seu livro "Álgebra", publicado em 1572, ao tratamento de regras para se operar com tais números. No final do século XVI, os matemáticos operavam com os números complexos segundo essas regras, mas o faziam com muita desconfiança, pois não os consideravam objetos matemáticos de fato, mas apenas como simples recurso de cálculo. Ainda assim, muitos progressos foram obtidos dessa forma [...] (NEVES, 2014, p. 03).
\end{abstract}

Para tanto, entre todos os matemáticos que contribuíram para a formulação dos Números Complexos, a presente pesquisa levou em consideração as definições apresentadas por William Rowan Hamilton (1805-1865), ao conceituar a estrutura formal da álgebra dos Números Complexos no plano como pares ordenados de Números Reais $(a, b)$.

Logo, este trabalho busca mostrar que, fazer uso de softwares educacionais livres para o ensino e aprendizagem de Matemática, pode ser uma forma diferenciada de tornar as aulas mais atrativas e dinâmicas. Visto que, tais softwares livres uma vez baixado nos dispositivos móveis como celulares, podem ser utilizados sem o uso da internet, possibilitando tato aos estudantes quanto professores uma ferramenta alternativa na construção de tabelas ou gráficos.

Posto isto, o objetivo deste trabalho é apresentar de forma breve o contexto histórico da formulação dos Números Complexos e dos Quatérnios, bem como utilizar o Software Educativo Livre GeoGebra e o Software Livre on-line QUATERNION, como recursos pedagógicos facilitando a compreensão das definições, propriedades e conceitos desses conjuntos.

\title{
O Conjunto dos Números Complexos e Quatérnios
}

O conjunto dos Números Complexos, denotado por $\mathbb{C}$, é definido como:

$$
\mathbb{C}=\left\{a+b i: a, b \in \mathbb{R} e i^{2}=-1\right\}
$$


A representação dos Números Complexos é da forma $z=a+b i$, em que $a$ e $b$ são números reais e $i$ a unidade imaginária. O conjunto dos Números Complexos é uma extensão do conjunto dos Números Reais, visto que compartilha das mesmas propriedades, munido das operações de adição e multiplicação, contendo uma estrutura de corpo algebricamente fechado. Na figura 1, observa-se as representações algébrica e geométrica dos Números Complexos consolidados por Hamilton, utilizando como recurso pedagógico o Software Educativo Livre GeoGebra. Segundo Soffa e Alcântara (2008, p. 4924 - 4925), "O que diferencia um software educativo de outras classes de softwares educacionais é o fato de ser desenvolvido com o desígnio de levar o aluno a construir um determinado conhecimento referente a um conteúdo didático". E ainda, segundo Silva (2019), os Softwares Educativos Livres são aqueles que não demandam custos.

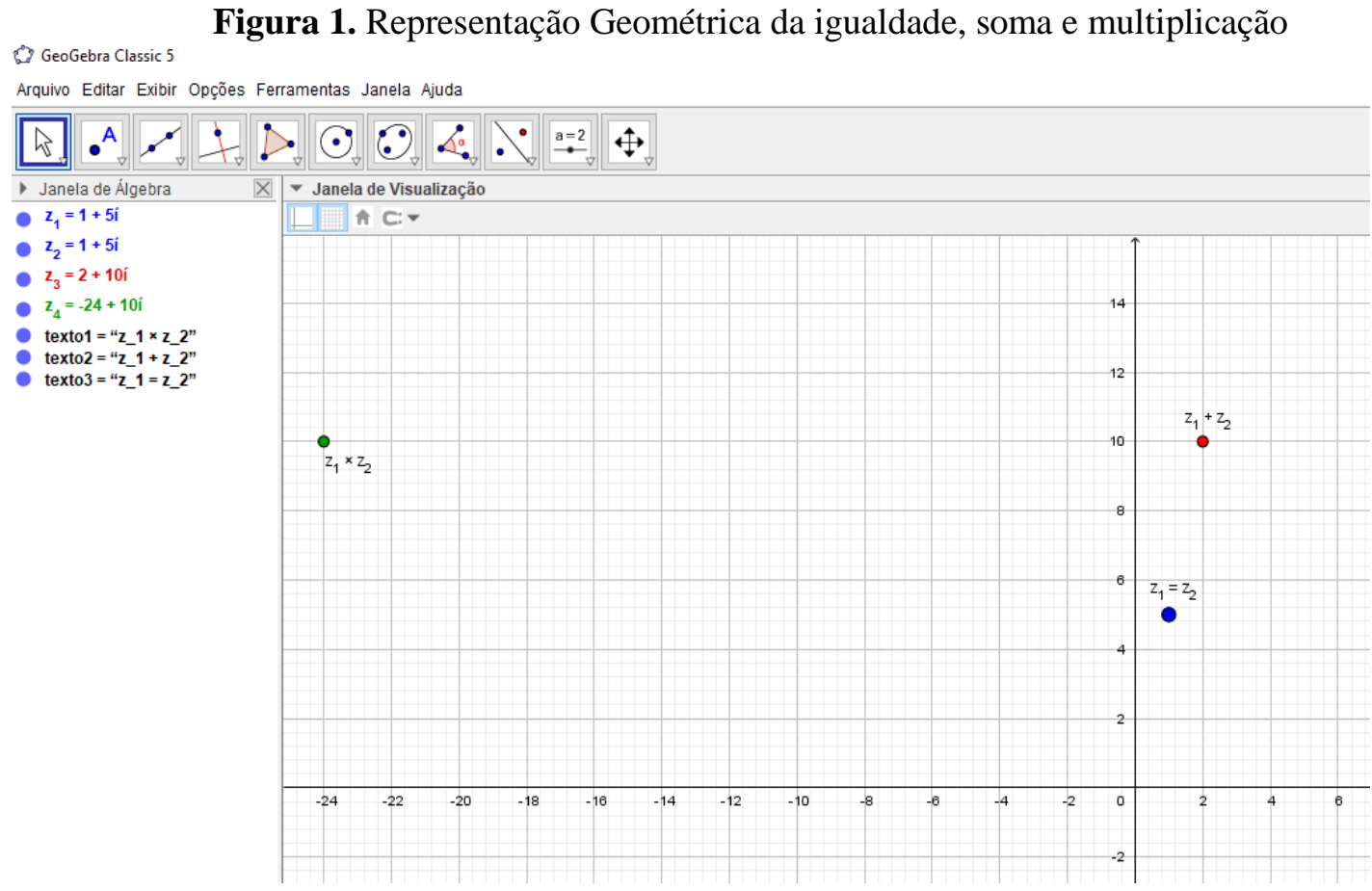

Fonte: Elaborada pelos autores.

Dados dois Números Complexos $z_{1}, z_{2}$ quaisquer, tais que $z_{1}=a+b i$ e $z_{2}=c+d i$, eles podem ser operados da seguinte maneira:

lgualdade

- $z_{1}=z_{2} \Leftrightarrow a=b$ e $c=d$

Adição 
- $z_{1}+z_{2}=(a+b i)+(c+d i)=(a+c)+(b+d) i$

Multiplicação

$z_{1} * z_{2}=(a+b i) *(c+d i)=(a c-b d)+(b c+a d) i$; em que $*$ indica o produto entre dois objetos (neste caso os complexos).

William Rowan Hamilton apresentou em 1833, na academia Irlandesa, um artigo sobre os números Complexos como pares ordenados de Números Reais $(a, b)$ no plano. Definiu as operações de soma e multiplicação de modo que: $(a, b)+(c, d)=(a+c, b+d)$ e $(a, b)(c, d)=(a c-b d, a d+b c)$, dessa forma, o par ordenado $(a, 0)$ representa um número real, e ainda, o par $(0,1)(0,1)=i^{2}=-1$, onde finalmente há uma explicação lógica para $\sqrt{-1}$ (GUEDES JUNIOR et al., 2016).

Dessa forma, houve a aceitação da álgebra dos Números Complexos pelos grandes matemáticos do século XIX, tornando possível realizar no plano transformações através das operações de adição determinando a translação, e a multiplicação determinando a rotação de corpos rígidos, sem alterar as propriedades do conjunto dos Números Reais. Neste contexto, segundo Neves (2008, p. 10), afirma que

Hamilton construiu uma estrutura algébrica para os "pares algébricos ou numéricos" $(x, y)$, similar a dos números complexos $x+i y$, de modo que tais números pudessem ser identificados pela relação ${ }^{4}(x, y)=(x, 0)+(0,1)(y, 0)=x+i y$. No final do artigo, Hamilton revelou que pretendia estender seus resultados em busca de uma "Teoria de Tripletos"5 $(x, y, z)$, já com a intenção de obter um modelo analítico para o espaço.

Passados anos, Hamilton estabeleceu, do ponto de vista prático, a solução desse problema que continuava sem respostas entre os matemáticos da época, onde em vez de três parâmetros ele passou a considerar quatro parâmetros para obter a rotação de objetos no espaço. Hamilton na tentativa de definir a multiplicação dos tripletos $(x, y, z)$, fazendo uso das mesmas propriedades dos pares numéricos $(x, y)$, se deparou com o fator $i j$ obtendo a não comutatividade nessa operação, a partir dessa manipulação algébrica surgiu um novo elemento $k=\sqrt{-1}$, que por sua vez é perpendicular simultaneamente a $i$ e $j$, estabelecendo a Álgebra dos Quatérnions (SANTOS, 2013).

Hamilton, quando caminhava pelo Canal Royal, em Dublin ao passar pela Ponte de Brougham, tirou seu canivete e deixou cravado em uma pedra a fórmula fundamental: $i^{2}=$

\footnotetext{
${ }^{4}$ Essa relação é encontrada nos livros didáticos de matemática do ensino médio.

${ }^{5}$ Hamilton usou a expressão "triplets". Por já ter sido consagrado nos textos em língua portuguesa, usaremos a expressão "tripletos" nesta dissertação.
} 
$j^{2}=k^{2}=i j k=-1$. Sir Willian Rowan Hamilton como era conhecido, mostrou pela primeira vez a utilização da classe dos Quatérnios para rotacionar corpos rígidos no espaço, em seu artigo "On Quatérnios", lido na Academia Real Irlandesa em 11 de novembro de 1844 (NEVES, 2008).

O conjunto dos Quatérnions, denotado por $\mathcal{H}$ em homenagem a Hamilton, é definido como:

$$
\mathcal{H}=\left\{a+b i+c j+d k ; a, b, c, d \in \mathbb{R} e i^{2}=j^{2}=k^{2}=i j k=-1\right\}
$$

Os quatérnios, no corpo dos Reais, com as operações de soma e multiplicação por escalar, formam um espaço vetorial. Portanto, transformações como rotacionar objetos podem ser feitas a partir das definições de alguns axiomas. Algebricamente é dizer que: $(\mathcal{H}, \mathbb{R},+, *)$. Ao realizar a soma ou multiplicação entre Quatérnions, e ainda, realizar a multiplicação entre um escalar e um Quatérnio se obtém um fechamento, isto é, os resultados das operações sempre pertencerão aos Quatérnios.

Para definir os axiomas do espaço vetorial, tem-se $\forall \alpha, \beta \in \mathbb{R} ; \forall q, q_{1}, q_{2} \in \mathcal{H}$.

Propriedades que satisfazem a adição;

Comutatividade: $q+q_{1}=q_{1}+q$;

Associatividade: $\left(q+q_{1}\right)+q_{2}=q+\left(q_{1}+q_{2}\right)$;

Vetor Nulo: $q+0=q$;

Existência de Elemento Inverso Aditivo: $q+(-q)=0$.

Propriedades que satisfazem a multiplicação;

Associatividade: $\left(q q_{1}\right) q_{2}=q\left(q_{1} q_{2}\right)$

Existência de Elemento Inverso Multiplicativo: $q q^{-1}=1$;

Existência de Elemento Neutro: $q * 1=q$.

Propriedades entre escalares e Quatérnios;

Associatividade: $q\left(\beta q_{1}\right)=(\beta q) q_{1}$;

Distributividade: $(\alpha+\beta) q=\alpha q+\beta q$;

Distributividade: $\left(q+q_{1}\right) \alpha=\alpha q+\alpha q_{1}$.

Igualdade entre Quatérnios.

Sejam $q=(a+b i+c j+d k)=(a, \vec{v}) \in \mathcal{H}$ e

$q_{1}=\left(a_{1}+b_{1} i+c_{1} j+d_{1} k\right)=\left(a_{1}, \overrightarrow{v_{1}}\right) \in \mathcal{H}$, então:

$$
q=q_{1} \Leftrightarrow\left(a=a_{1}\right) \text { e }\left(\vec{v}=\overrightarrow{v_{1}}\right) .
$$




\section{ENSIN@UFMS 2021}

ISSN 2525-7056

\section{Adição}

Para realizar a adição com os Quatérnios, deve-se proceder igualmente como nos Números Complexos, somando as coordenadas correspondentes.

Sejam $q=(a+b i+c j+d k)=(a, \vec{v}) \in \mathcal{H}$ e $q_{1}=\left(a_{1}+b_{1} i+c_{1} j+d_{1} k\right)=\left(a_{1}, \overrightarrow{v_{1}}\right) \in$ $\mathcal{H}$, então:

$q+q_{1}=(a+b i+c j+d k)+\left(a_{1}+b_{1} i+c_{1} j+d_{1} k\right)=\left(a+a_{1}\right)+\left(b+b_{1}\right) i+\left(c+c_{1}\right) j+$ $\left(d+d_{1}\right) k$.

\section{Multiplicação}

Realizar a multiplicação dos Quatérnios, torna-se fácil quando se aplica inicialmente a representação gráfica e algébrica conforme ilustram as figuras 8 e 9 (não comutatividade desse corpo), bem como as relações fundamentais descrita na definição 1.

Figura 2. Representação Gráfica da não Comutatividade da Multiplicações de Quatérnios.

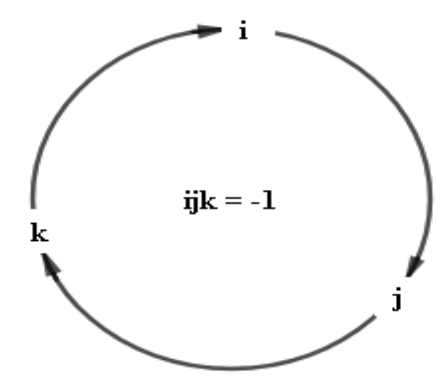

Fonte: Elaborada pelos autores.

Tabela 1 Representação Gráfica da não Comutatividade da Multiplicações de Quatérnios.

Fonte: Elaborada pelos autores

\begin{tabular}{c|c|c|c|c|}
\hline & 1 & $\mathrm{i}$ & $\mathrm{j}$ & $\mathrm{k}$ \\
\hline $\mathrm{1}$ & $1 \cdot 1=1$ & $1 \cdot i=i$ & $1 \cdot j=j$ & $1 \cdot k=k$ \\
\hline $\mathrm{i}$ & $i \cdot 1=i$ & $i \cdot i=-1$ & $i \cdot j=k$ & $i \cdot k=-j$ \\
\hline $\mathrm{j}$ & $j \cdot 1=j$ & $j \cdot i=-k$ & $j \cdot j=-1$ & $j \cdot k=i$ \\
\hline $\mathrm{k}$ & $k \cdot 1=k$ & $k \cdot i=j$ & $k \cdot j=-i$ & $k \cdot k=-1$ \\
\hline
\end{tabular}

Para realizar a multiplicação dos Quatérnios, as seguintes relações fundamentais foram definidas.

Definição 1:

- $\quad i j=k \Rightarrow i i j=i k \Rightarrow i^{2} j=i k \Rightarrow(-1) j=i k \Rightarrow i k=-j$ 
- $j k=i \Rightarrow j j k=j i \Rightarrow j^{2} k=j i \Rightarrow(-1) k=j i \Rightarrow j i=-k$

- $k i=j \Rightarrow k k i=k j \Rightarrow k^{2} i=k j \Rightarrow(-1) i=k j \Rightarrow k j=-i$

- $\quad i j=k \Rightarrow i j k=k k \Rightarrow i j k=k^{2} \Rightarrow i j k=-1$

Desta forma, a multiplicação entre Quatérnios é dada por:

Sejam $q=(a+b i+c j+d k)=(a, \vec{v}) \in \mathcal{H}$ e $q_{1}=\left(a_{1}+b_{1} i+c_{1} j+d_{1} k\right)=\left(a_{1}, \overrightarrow{v_{1}}\right) \in$ $\mathcal{H}$, então:

$q q_{1}=(a+b i+c j+d k)\left(a_{1}+b_{1} i+c_{1} j+d_{1} k\right)=a\left(a_{1}+b_{1} i+c_{1} j+d_{1} k\right)+b i\left(a_{1}+b_{1} i+\right.$ $\left.c_{1} j+d_{1} k\right)+c j\left(a_{1}+b_{1} i+c_{1} j+d_{1} k\right)+d k\left(a_{1}+b_{1} i+c_{1} j+d_{1} k\right)=a a_{1}+a b_{1} i+a c_{1} i+$ $a d_{1} k+b i a_{1}+b i b_{1} i+b i c_{1} j+b i d_{1} k+c j a_{1}+c j b_{1} i+c j c_{1} j+c j d_{1} k+d k a_{1}+d k b_{1} i+$ $d k c_{1} j+d k d_{1} k=a a_{1}+b b_{1} i^{2}+c c_{1} j^{2}+d d_{1} k^{2}+a b_{1} i+a c_{1} j+a d_{1} k+b a_{1} i+c a_{1} j+d a_{1} k+$ $b c_{1} i j+b d_{1} i k+c b_{1} j i+c d_{1} j k+d b_{1} k i+d c_{1} k j=a a_{1}-\left(b b_{1}+c c_{1}+d d_{1}\right)+a\left(b_{1} i+c_{1} j+\right.$ $\left.d_{1} k\right)+a_{1}(b i+c j+d k)+b c_{1} k-b d_{1} j-c b_{1} k+c b_{1} i+d b_{1} j-d c_{1} i=a a_{1}-\left(b b_{1}+c c_{1}+\right.$ $\left.d d_{1}\right)+a\left(b_{1} i+c_{1} j+d_{1} k\right)+a_{1}(b i+c j+d k)+\left(c d_{1}-d c_{1}\right) i+\left(d b_{1}-b d_{1}\right) j+\left(b c_{1}-c b_{1}\right) k$. $q q_{1}=a a_{1}-\left(b b_{1}+c c_{1}+d d_{1}\right)+a\left(b_{1} i+c_{1} j+d_{1} k\right)+a_{1}(b i+c j+d k)+\left(c d_{1}-d c_{1}\right) i+$ $\left(d b_{1}-b d_{1}\right) j+\left(b c_{1}-c b_{1}\right) k$.

Os resultados obtidos em (1), podem ser expressos em termos de produtos escalares e vetoriais.

- $a a_{1}-\left(b b_{1}+c c_{1}+d d_{1}\right)=a a_{1}-\vec{v} \cdot \overrightarrow{v_{1}}$

- $a\left(b_{1} i+c_{1} j+d_{1} k\right)+a_{1}(b i+c j+d k)=a \overrightarrow{v_{1}}+a_{1} \vec{v}$

- $\left(c d_{1}-d c_{1}\right) i+\left(d b_{1}-b d_{1}\right) j+\left(b c_{1}-c b_{1}\right) k=\vec{v} \times \overrightarrow{v_{1}}$

- $q q_{1}=\left(a a_{1}-\vec{v} \cdot \overrightarrow{v_{1}}\right)+\left(a \overrightarrow{v_{1}}+a_{1} \vec{v}+\vec{v} \times \overrightarrow{v_{1}}\right)$; em que $\cdot$ indica o produto escalar $\mathrm{e} \times 0$ produto vetorial.

Norma de um Quatérnio

Seja $q=(a+b i+c j+d k)=(a, \vec{v}) \in \mathcal{H}$, define-se a norma ou valor absoluto de $q$, sendo o número não negativo, como segue: $\|q\|=\sqrt{q \bar{q}}=\sqrt{a^{2}+b i^{2}+c j^{2}+d k^{2}}=$ $\sqrt{a^{2}+|\vec{v}|^{2}}$

Conjugado

Seja $q=(a+b i+c j+d k)=(a, \vec{v}) \in \mathcal{H}$, é definido o conjugado de $q$, como: $\bar{q}=$ $(a-b i-c j-d k)=(a,-\vec{v}) \in \mathcal{H}$.

Proposição 1: A relação entre o módulo e conjugado dos Quatérnios é dado por: $q \bar{q}=\bar{q} q$ 
Sejam $q=(a+b i+c j+d k)=(a, \vec{v}) \in \mathcal{H} \mathrm{e}$

$\bar{q}=(a-b i-c j-d k)=\left(a_{1},-\overrightarrow{v_{1}}\right) \in \mathcal{H}$, então:

$q \bar{q}=(a+b i+c j+d k)(a-b i-c j-d k)=(a a-a b i-a c j-a d k)+\left(-a b i-b^{2} i^{2}-\right.$

$b c i j-b d i k)+\left(-a c j-b c j i-c^{2} j^{2}-c d j k\right)+\left(-a d k-b d k i-c d k j-d^{2} k^{2}\right)=\left[a^{2}+(a b i-\right.$

$a b i)+(a c j-a c j)+(a d k-a d k)]+\left[-b^{2}(-1)-c^{2}(-1)-d^{2}(-1)\right]+[(b c k-b c k)+(b d j-$ $b d j)+(c d i-c d i)]=a^{2}+b^{2}+c^{2}+d^{2}$.

$\bar{q} q=(a+b i+c j+d k)(a-b i-c j-d k)=(a a-a b i-a c j-a d k)+\left(-a b i-b^{2} i^{2}-\right.$

$b c i j-b d i k)+\left(-a c j-b c j i-c^{2} j^{2}-c d j k\right)+\left(-a d k-b d k i-c d k j-d^{2} k^{2}\right)=\left[a^{2}+(a b i-\right.$

$a b i)+(a c j-a c j)+(a d k-a d k)]+\left[-b^{2}(-1)-c^{2}(-1)-d^{2}(-1)\right]+[(b c k-b c k)+(b d j-$ $b d j)+(c d i-c d i)]=a^{2}+b^{2}+c^{2}+d^{2}$.

Portanto,

$\|q\|^{2}=q \bar{q}=\bar{q} q=a^{2}+b^{2}+c^{2}+d^{2}$.

Proposição 2: Relações entre dois Quatérnios conjugados:

$\overline{q q_{1}}=\bar{q} \overline{q_{1}}$

Sejam $q=(a+b i+c j+d k)=(a, \vec{v}) \in \mathcal{H} \mathrm{e}$

$q_{1}=\left(a_{1}+b_{1} i+c_{1} j+d_{1} k\right)=\left(a_{1}, \overrightarrow{v_{1}}\right) \in \mathcal{H}$, então:

$q q_{1}=(a+b i+c j+d k)\left(a_{1}+b_{1} i+c_{1} j+d_{1} k\right)$.

$q q_{1}=\left(a a_{1}-b b_{1}-c c_{1}-d d_{1}\right)+\left(a b_{1}+b a_{1}+c d_{1}-d c_{1}\right) i+\left(a c_{1}-b d_{1}+c a_{1}+d b_{1}\right) j+$

$\left(a d_{1}+b c_{1}-c b_{1}+d a_{1}\right) k$.

$\overline{q q_{1}}=\left(a a_{1}-b b_{1}-c c_{1}-d d_{1}\right)-\left(a b_{1}+b a_{1}+c d_{1}-d c_{1}\right) i-\left(a c_{1}+b d_{1}-c a_{1}-d b_{1}\right) j-$

$\left(a d_{1}+b c_{1}-c b_{1}+d a_{1}\right) k$.

$\bar{q} \overline{q_{1}}=(a-b i-c j-d k)\left(a_{1}-b_{1} i-c_{1} j-d_{1} k\right)$.

$\bar{q} \overline{q_{1}}=\left(a a_{1}-b b_{1}-c c_{1}-d d_{1}\right)+\left(-a b_{1}-b a_{1}-c d_{1}+d c_{1}\right) i+\left(-a c_{1}+b d_{1}-c a_{1}-d b_{1}\right) j+$ $\left(-a d_{1}-b c_{1}+c b_{1}-d a_{1}\right) k$.

$\bar{q} \overline{q_{1}}=\left(a a_{1}-b b_{1}-c c_{1}-d d_{1}\right)-\left(a b_{1}+b a_{1}+c d_{1}-d c_{1}\right) i-\left(a c_{1}+b d_{1}-c a_{1}-d b_{1}\right) j-$ $\left(a d_{1}+b c_{1}-c b_{1}+d a_{1}\right) k$.

\section{Quatérnios Unitários}

Seja $q \in \mathcal{H}$, um Quatérnio unitário ou normalizado $\|q\|=1$, a inversa é o complexo conjugado: 


$$
q^{-1}=\bar{q}
$$

Quatérnios Inversos

Todo Quatérnio diferente de zero tem um multiplicativo inverso, que é definido como:

$$
q^{-1} q=q q^{-1}
$$

Considerando $q^{-1}$ um Quatérnio inverso à direita, e assim, observa-se que para normalizar um Quatérnio basta fazer a razão entre o Quatérnio e seu módulo ao quadrado. Assim:

$$
q q^{-1}=\frac{q \bar{q}}{\|q\|^{2}}=\frac{\|q\|^{2}}{\|q\|^{2}}=1
$$

Ao considerar $q^{-1}$ um Quatérnio inverso à esquerda, segue:

$$
q^{-1} q=\frac{\bar{q} q}{\|q\|^{2}}=\frac{\|q\|^{2}}{\|q\|^{2}}=1
$$

Divisão entre Quatérnios

Sejam $q=(a+b i+c j+d k)=(a, \vec{v}) \in \mathcal{H} \mathrm{e}$ $q_{1}=\left(a_{1}+b_{1} i+c_{1} j+d_{1} k\right)=\left(a_{1}, \overrightarrow{v_{1}}\right) \in \mathcal{H}$, é realizada a divisão entre $q$ e $q_{1}$, como:

Divisão a esquerda:

$$
\frac{q}{q_{1}}=q_{1}^{-1} q
$$

Divisão a direita:

$$
\frac{q}{q_{1}}=q q_{1}^{-1}
$$

\section{Rotação com Quatérnios}

Operando um ponto no $\mathbb{R}^{3}$, representado por um vetor $\vec{r}=\left(r_{x}, r_{y}, r_{z}\right)$, e, transformando esse vetor num Quatérnio $q \operatorname{com} q \in \mathcal{H}$, tem-se que a parte escalar desse Quatérnio será nula obtendo um Quatérnio puro $p=(0, r)$. A rotação anti-horária de um ângulo $\theta$, que será aplicado no eixo definido pelo vetor unitário $\vec{n}=\left(n_{x}, n_{y}, n_{z}\right)$, é fundamental ser representada por um Quatérnio unitário. 
Figura 3. Eixos de Rotação

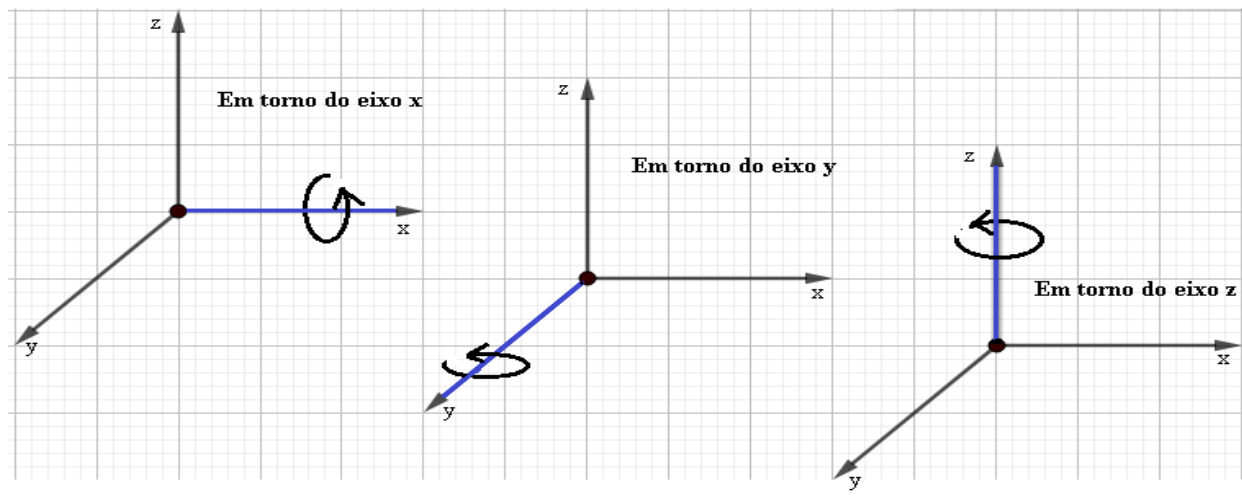

Fonte: Elaborada pelos autores.

Serão utilizado as Identidades do Cálculo Vetorial:

$$
\begin{gathered}
\vec{v} \cdot(\vec{r} \times \vec{v})=0 \\
s \vec{v} \times \vec{r}=-s \vec{r} \times \vec{v} \\
\vec{v} \times(\vec{v} \times \vec{r})=(\vec{v} \cdot \vec{r}) \vec{v}-(\vec{v} \cdot \vec{v}) \vec{r}
\end{gathered}
$$

A expressão de rotação de um Quatérnio pode ser descrita como:

$q p \bar{q}=(s, \vec{v})(0, \vec{r})(s,-\vec{v})=(s, \vec{v})(0 s-\vec{r}(-\vec{v}),-0 \vec{v}+s \vec{r}+\vec{r} \times-\vec{v}=(s, \vec{v})(\vec{r} \cdot \vec{r}, s \vec{r}-\vec{r} \times \vec{v})=$ $(s(\vec{r} \cdot \vec{r})-\vec{v} \cdot(s \vec{r}-\vec{r} \times \vec{v}), s(s \vec{r}-\vec{r} \times \vec{v})+(\vec{r}, \vec{v}) \vec{v}+\vec{v} \times(s \vec{r}-\vec{r} \times \vec{v}))=(s(\vec{r} \cdot \vec{r})-\vec{v} \cdot s \vec{r}-$ $\left.\vec{v} \cdot(-\vec{r} \times \vec{v}), s^{2} \vec{r}-s \vec{r} \times \vec{v}+(\vec{r} \cdot \vec{v}) \vec{v}+\vec{v} \times s \vec{r}+\vec{v} \times(-\vec{r} \times \vec{v})\right)=(s(\vec{r} \cdot \vec{r})-s(\vec{r} \cdot \vec{v})+\vec{v} \cdot(\vec{r} \cdot$ $\left.\vec{v}), s^{2} \vec{r}+s \vec{v} \times \vec{r}+(\vec{r} \cdot \vec{v}) \vec{v}+s \vec{v} \times \vec{r}+\vec{v} \times(\vec{v} \times \vec{r})\right)=\left(\vec{v} \cdot(\vec{r} \times \vec{v}), s^{2} \vec{r}+(\vec{r} \cdot \vec{v}) \vec{v}+2 s \vec{v} \times \vec{r}+\right.$ $(\vec{v} \cdot \vec{r}) \vec{v}-(\vec{v} \cdot \vec{v}) \vec{r})=\left(0, s^{2} \vec{r}-(\vec{v} \cdot \vec{v}) \vec{r}+2(\vec{v} \cdot \vec{r}) \vec{v}+2 s \vec{v} \times \vec{r}\right)$

Por definição, segue:

$$
\begin{aligned}
q & =\left(\cos \frac{1}{2} \theta, \operatorname{sen} \frac{1}{2} \theta \vec{n}\right) \\
\bar{q} & =\left(\cos \frac{1}{2} \theta,-\operatorname{sen} \frac{1}{2} \theta \vec{n}\right)
\end{aligned}
$$

Ao realizar uma rotação no espaço é necessário fazer uma interpretação geométrica. Sendo $q=(s, \vec{v})$ um vetor unitário, segue pela identidade fundamental da trigonometria, que existe um $\theta$ tal que $s=\cos \theta,\|v\|=\operatorname{sen} \theta$ e $\vec{n}=\left(n_{x}, n_{y}, n_{z}\right) \Leftrightarrow\|\vec{n}\|=1$ onde o vetor $\vec{v}$ é um vetor diretor que representa uma rotação no espaço.

Por definição, temos:

$$
q=(s, \vec{v})=(\cos \theta, \operatorname{sen} \theta \vec{n})
$$




\section{ENSIN@UFMS 2021}

ISSN 2525-7056

Dessa forma, substitui-se essa interpretação geométrica na expressão obtida em (2), segue:

$$
\begin{gathered}
s=\cos \theta \Rightarrow s^{2}=\cos ^{2} \theta \\
\vec{v}=\operatorname{sen} \theta \vec{n}
\end{gathered}
$$

$q p \bar{q}=\left(0, s^{2} \vec{r}-(\vec{v} \cdot \vec{v}) \vec{r}+2(\vec{v} \cdot \vec{r}) \vec{v}+2 s \vec{v} \times \vec{r}\right)=\left(0,\left(\cos ^{2} \theta\right) \vec{r}-(\operatorname{sen} \theta \vec{n} \cdot \operatorname{sen} \theta \vec{n}) \vec{r}+\right.$ $2(\operatorname{sen} \theta \vec{n} \cdot \vec{r}) \operatorname{sen} \theta \vec{n}+2 \cos \theta(\operatorname{sen} \theta \vec{n}) \times \vec{r})=\left(0,\left(\cos ^{2} \theta\right) \vec{r}-\left(\operatorname{sen}^{2} \theta \vec{n} \cdot \vec{n}\right) \vec{r}+\left(2 \operatorname{sen}^{2} \theta\right)(\vec{n}\right.$. $\vec{r}) \vec{n}+(2 \cos \theta \operatorname{sen} \theta) \vec{n} \times \vec{r})=\left(0,\left(\cos ^{2} \theta\right) \vec{r}-\left(\operatorname{sen}^{2} \theta\right) \vec{r}+(1-\cos 2 \theta)(\vec{n} \cdot \vec{r}) \vec{n}+(\operatorname{sen} 2 \theta) \vec{n} \times\right.$ $\left.\vec{r})=\left(0,\left(\cos ^{2} \theta-\operatorname{sen}^{2} \theta\right) \vec{r}+(1-\cos 2 \theta)(\vec{n} \cdot \vec{r}) \vec{n}+(\operatorname{sen} 2 \theta) \vec{n} \times \vec{r}\right)\right)=(0,(\cos 2 \theta) \vec{r}+(1-$ $\cos 2 \theta)(\vec{n} \cdot \vec{r}) \vec{n}+(\operatorname{sen} 2 \theta) \vec{n} \times \vec{r})$.

É possível observar que dado um vetor $\vec{n}$ unitário e um ângulo de rotação $\theta$, o Quatérnio $q=(\cos \theta, \operatorname{sen} \theta \vec{n})$ executa uma rotação de $p$ em torno do eixo $\vec{n}$ de ângulo $2 \theta$. Ao multiplicar dois Quatérnios unitários de eixos e ângulos diferentes, naturalmente é possível representar a composição de suas rotações, ocorrendo a parametrização entre os quatérnios. Então, a rotação composta pode ser realizada da seguinte forma:

$$
q_{4}=\overline{q_{3}} \Rightarrow q_{3} p q_{4}=q_{3} p \bar{q}_{3}=R q_{3}(p)
$$

Conclui-se que:

$$
\begin{aligned}
& q_{3}=q_{1} q_{2}=\left(s_{2}, \vec{v}_{2}\right)\left(s_{1}, \vec{v}_{1}\right)=\left(s_{2} s_{1}-\vec{v}_{2} \vec{v}_{1}, s_{2} \vec{v}_{1}+s_{1} \vec{v}_{2}+\vec{v}_{2} \times \vec{v}_{1}\right) \Leftrightarrow \bar{q}_{3}=\left(s_{2} s_{1}-\right. \\
& \left.\vec{v}_{2} \vec{v}_{1},-s_{2} \vec{v}_{1}-s_{1} \vec{v}_{2}-\vec{v}_{2} \times \vec{v}_{1}\right)=\left(s_{2} s_{1}-\vec{v}_{2} \vec{v}_{1},-s_{2} \vec{v}_{1}-s_{1} \vec{v}_{2}+\vec{v}_{2} \times \vec{v}_{1}\right) \Leftrightarrow \overline{q_{3} q_{2}}= \\
& \left(s_{1},-\vec{v}_{1}\right)\left(s_{2},-\vec{v}_{2}\right)=\bar{q}_{3}=q_{4}=\left(s_{2} s_{1}-\vec{v}_{2} \vec{v}_{1},-s_{2} \vec{v}_{1}-s_{1} \vec{v}_{2}+\vec{v}_{2} \times \vec{v}_{1}\right) .
\end{aligned}
$$

A primeira rotação pode ser representada pelo Quatérnio:

$$
q_{1}=\left(\cos \frac{180^{\circ}}{2}, \operatorname{sen} \frac{180^{\circ}}{2}(0,0,1)\right)=\left(\cos 90^{\circ}, \operatorname{sen} 90^{\circ}(0,0,1)\right)=(0,(1)(0,0,1))=(0,(0,0,1))
$$


Figura 4. Rotação via Quatérnios representado no Software Livre on-line Quaternion - Vista Superior.

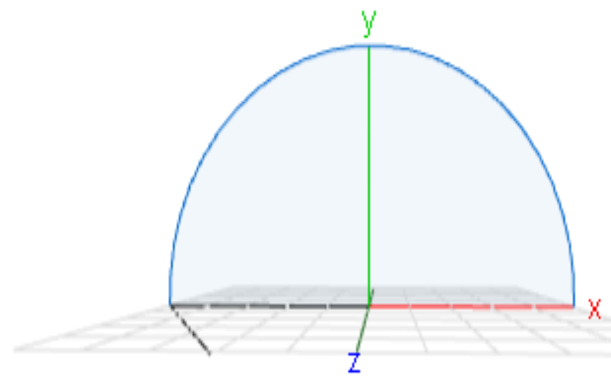

Quaternion

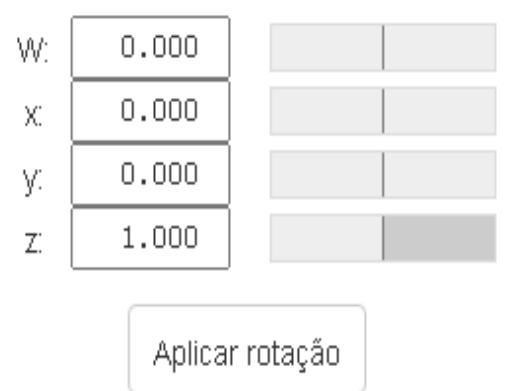

Fonte: Acervo dos autores.

A segunda rotação é dada pelo Quatérnio:

$q_{2}=\left(\cos \frac{180^{\circ}}{2}, \operatorname{sen} \frac{180^{\circ}}{2}(1,0,0)\right)=\left(\cos 90^{\circ}, \operatorname{sen} 90^{\circ}(1,0,0)\right)=(0,(1)(1,0,0))=(0,(1,0,0))$

Figura 5. Rotação via Quatérnios representado no Software Livre on-line QUATERNION - Vista Superior.

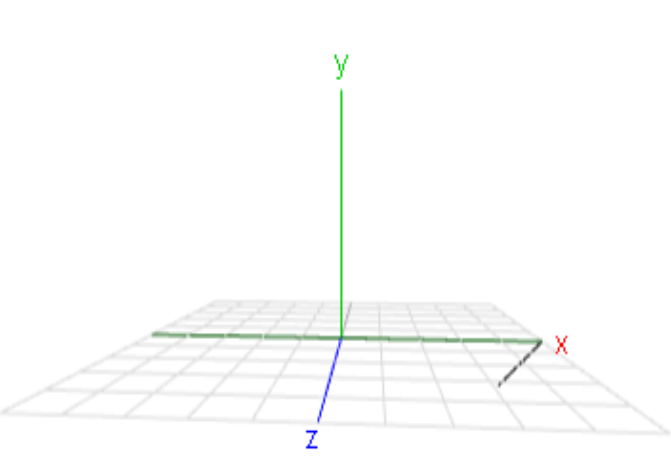

Quaternion

Fonte: Acervo dos autores.

Para saber o comportamento algébrico do corpo rígido em seu instante inicial e após às duas rotações, suponha-se que o ponto inicial ocupe a posição $\vec{v}_{1}=(10,5,10)$, ou seja, 10 na direção das abscissas, 5 na direção das ordenadas e 10 na direção das cotas. Aplicase a rotação na posição inicial sobre o ponto $\vec{v}_{1}=(10,5,10)$ a posição final será $\vec{v}_{2}=$ $(-10,5,-10)$. Assim, ao multiplicar dois quatérnio, pode-se encontrar a parametrização em coordenadas, determinando a posição de um corpo rígido no espaço e sua trajetória. 
$q_{3}\left(0, \vec{p}_{1}\right) \bar{q}_{3}=(0,(0,-1,0)),(0,(10,5,10)(0,-(0,-1,0)=(0,(0,-1,0))(0 \cdot 0+$

$(0,-1,0)(10,5,10), 0(10,5,10)+0(-(0,-1,0)+(0,1,0) \times(10,5,10)=(0,(0,-1,0)),(0+(0-$ $5+0),(10,0-10)=(0,(0,-1,0)(-5,(10,0,-10)=(-5 \cdot 0-$

$(10,0,-10)(0,1,0),-5(0,-1,0)+0(10,0,-10)+(10,0,-10) \times(0,-1,0)=(0-(10 \cdot 0+0 \cdot$

$1-10 \cdot 0),(0,5,0)+(-10,0,-10)=(0,(-10,5,-10))$.

Ao considerar o ponto $A=\vec{v}_{1}=(10,5,10)$ em um corpo rígido qualquer, e utilizando o Software ${ }^{6}$ GeoGebra; nas figuras seguintes é possível visualizar a rotação deste mesmo corpo representado pela rotação composta via quatérnios do ponto $A=\vec{v}_{1}=(10,5,10)$ contido na esfera. Traça-se um arco de círculo máximo até o ponto $B=\vec{v}_{2}=(-10,5,-10)$, é possível notar a translação ocorrida do ponto $A$ até o ponto $B$.

Figura 6. Rotação via Quatérnio representado no Software GeoGebra 3D - Vista Frontal.

Fonte: Elaborada pelos autores

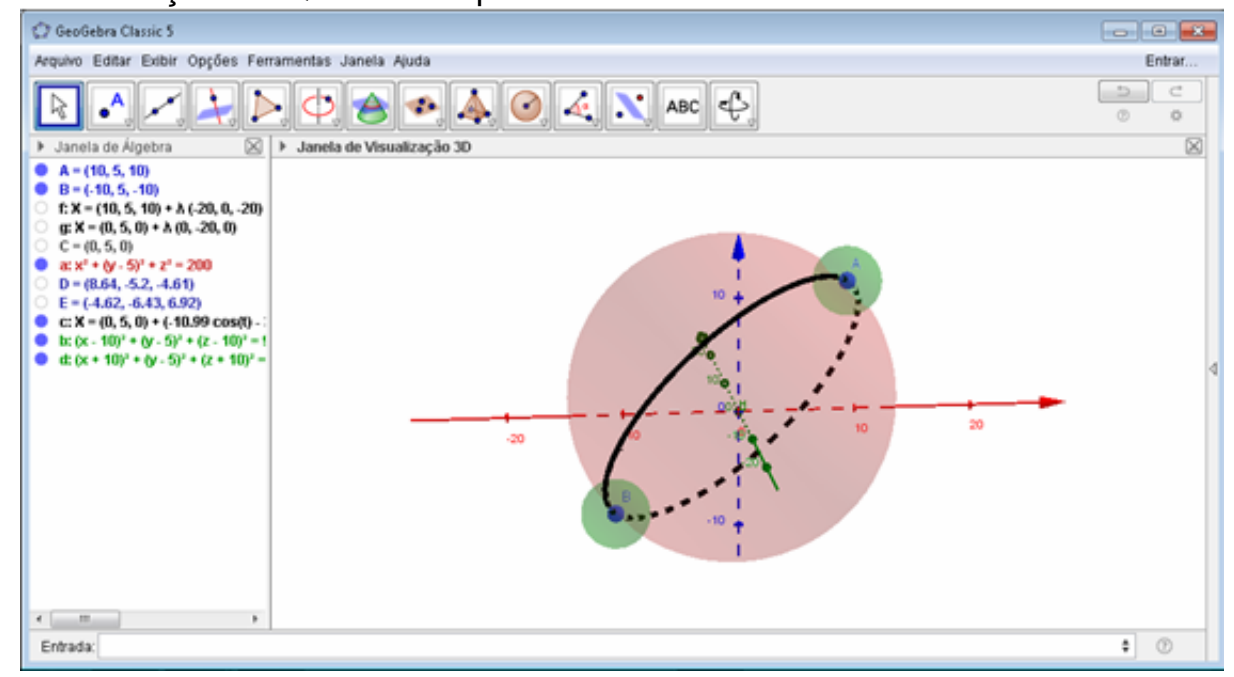

\footnotetext{
${ }^{6}$ Para facilitar o desenvolvimento do estudo utilizaremos Software com o mesmo significado de Softwares Educativos Livre ou Softwares Livre on-line conforme mencionado no corpo de texto.
} 


\section{ENSIN@UFMS 2021}

ISSN 2525-7056

Figura 7. Rotação via Quatérnio representado no Software GeoGebra 3D - Vista Superior-Posterior.

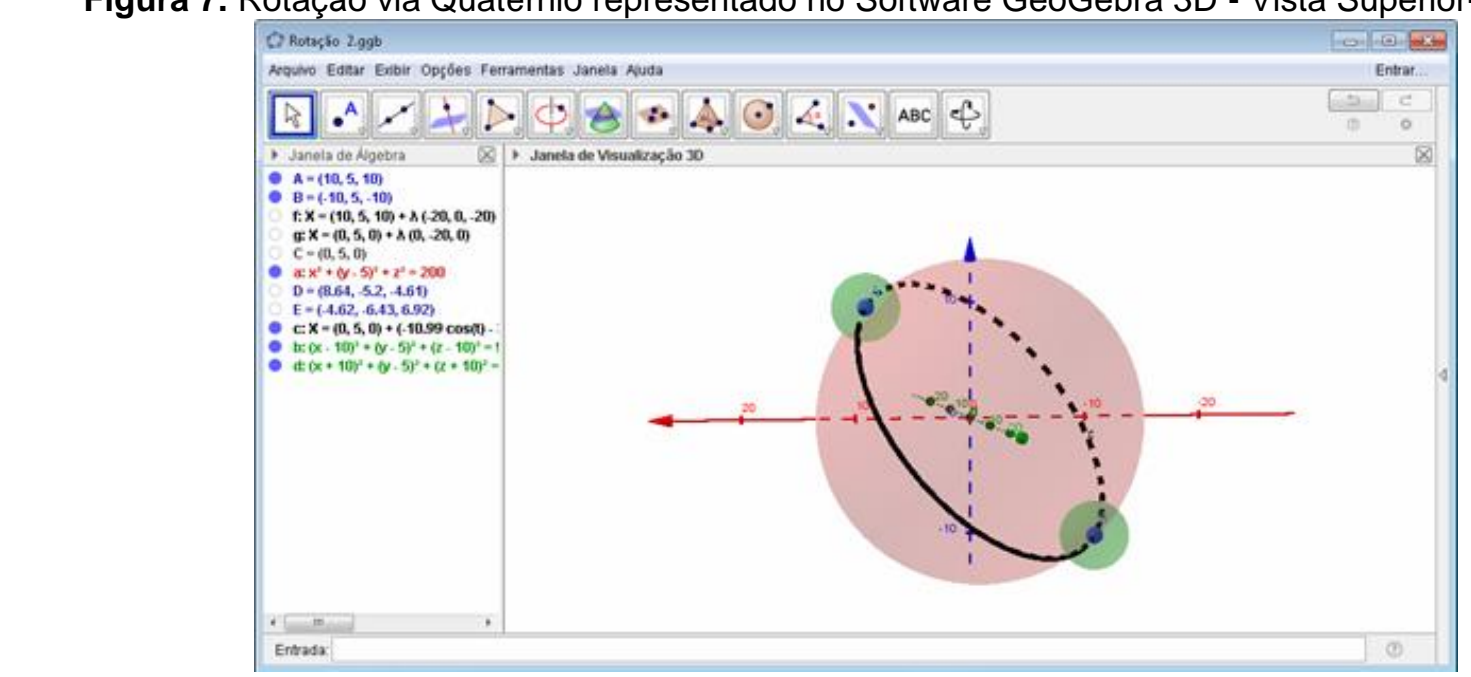

Fonte: Elaborada pelos autores

Dessa forma, a multiplicação entre Quatérnios facilita a parametrização de objetos em coordenadas de maneira simples, com composição de números arbitrários de rotação, através de quaisquer pontos desse objeto. Então, para representar a orientação de um corpo rígido basta tomar um Quatérnio.

\section{Ensino de matemática utilizando tecnologia e os uso dos softwares livres GeoGebra e Quaternion}

As Tecnologias Educacionais, em especial os Softwares Educativo Livre como o GeoGebra e QUATERNION conforme mostra a presente pesquisa, podem facilitar o entendimento de conteúdos matemáticos abstratos tornando as aulas mais dinâmicas, ágeis e prazerosas. Segundo Azevedo Silva, Pereira e Souza (2018, p. 218), “[...] a metodologia utilizada no desenvolvimento e aplicação, facilitará o alcance do objetivo principal, possibilitando a representação dos números complexos de maneira tecnológica e diferenciada".

Nesta mesma perspectiva, Soffa e Alcântara (2008, p. 3 - 4), afirmam que o Software Educativo é "[...] desenvolvido com o desígnio de levar o aluno a construir um determinado conhecimento referente a um conteúdo didático. O objetivo de um software educativo é a de favorecer os processos de ensino-aprendizagem e sua característica principal é seu caráter didático". E ainda, os autores relatam que: 
A escolha do software, além de ser fundamental para o trabalho que o educador irá desenvolver com seus alunos, pressupõe uma visão de mundo, uma concepção de educação. Fica assim evidenciada a importância que deve ser dada à escolha dos programas que serão selecionados para serem utilizados com intuito educacional (SOFFA; ALCÂNTARA, 2008, p. 4).

Dessa forma, em consonância com os autores acima, segundo Gomes et al. (2002), ao ensinar conteúdos Matemáticos fazendo uso de Softwares afirma que:

Assim, a aprendizagem matemática através de softwares deve ser baseada em situações-problema que considerem: os processos cognitivos, o raciocínio, as estratégias adotadas durante 0 processo de resolução, os estágios de desenvolvimento relativos às habilidades envolvidas e caracterização dos diversos problemas e seu nível de complexidade (GOMES et al., 2002, p. 5).

O uso desses Softwares, possibilitará a visualização e a representação algébrica e geométrica das operações dessas classes de números imaginários, podendo propiciar uma melhor percepção da rotação no plano que está relacionada diretamente com os Números Complexos e nas transformações lineares/rotações no espaço que está associada aos Quatérnios, operando de forma conjunta a Álgebra e a Geometria. Para Silva (2019, p. 97), "E, em se tratando de Softwares Livres, não há necessidade de ficar conectado à Internet para acessar e utilizar o software, uma vez baixado, o uso é independente da navegação na rede." Nestas circunstâncias, durante a formação inicial e/ou continuada dos professores de Matemática, é fundamental que haja a familiarização das novas Tecnologias Educacionais. Neste aspecto as Diretrizes Nacionais de Educação determinam que:

[...]curso e licenciado devem adquirir familiaridade com o uso do computador como instrumento de trabalho, incentivando-se sua utilização para o ensino de matemática, em especial para formação e soluções de problemas. É importante também a familiarização do licenciado, ao longo do curso, com outras tecnologias que possa contribuir para o ensino de Matemática (PARECER CNE/CES, 2001, p. 06).

Em vista disso, há uma necessidade de restruturação no método tradicionalista de ensinar conteúdo usando somente, lápis, giz e quadro dentro da sala de aula, durante a formação inicial dos professores. Neste contexto, segundo Ferreira (2012), "[...] é relevante repensar a formação dos professores de forma ampla, a fim de caminharmos para processos educativos mais integrados, conectados e interativos" (p. 5).

As novas tecnologias educacionais como proposta pedagógica, exigem uma formação docente bastante ampla. Visto que as ações pedagógicas não envolvem a penas a utilização do computador ou de um software específico, mas exige que se trabalhe com técnicas que incentivem a participação dos alunos, a interação 


\section{ENSIN@UFMS 2021}

ISSN 2525-7056

entre eles e a produção do conhecimento, ou seja, envolve muito mais do que apreender conhecimentos digitais. (FERREIRA, 2012, p. 10).

Para tanto, o ensino dos Números Complexos e dos Quatérnios à luz das Novas Tecnologias em especial do Software Educativo Livre GeoGebra e o Software Livre on-line QUATERNION, possibilitam aos alunos uma melhor compreensão algébrica e geométrica desses números imaginários, bem como, melhora a interação entre (alunos e alunos, professores e alunos). Aprimora o pensamento investigativo, levando o aluno a buscar novas soluções aos problemas propostos.

Criado por Markus Hohenwarter o Software GeoGebra é um programa livre e gratuito com a finalidade de apoiar o contexto escolar dentro da sala de aula (HOHENWARTER, 2016). O software, consiste numa dinâmica onde combina conceitos geométricos e algébricos ao passo que o objeto matemático é construído, propiciando de maneira ágil e instantânea a visualização das propriedades e aplicações matemáticas, bem como promove a interação individual e/ou coletiva do aluno coma novas tecnologias educacionais.

O Software QUATERNION, é um programa on-line livre e gratuito, no qual, mostra a transformação linear graficamente de objetos de dimensão quatro (GALLENBERGER, 2021). Esse (o QUATERNION), trabalha com os conceitos da álgebra e geometria em uma única tela, permitindo que o aluno perceba a representação geométrica ao passo que insere os comandos algébricos. Dessa forma, possibilita o conhecimento matemático diferenciado aumentando a criatividade do aluno através de uma linguagem de fácil compreensão, bem como facilita o processo de ensino e aprendizagem dos envolvidos.

\section{Pesquisa com alunos de Licenciatura em Matemática sobre uso de Tecnologias no ensino de Números Complexos e Quatérnios}

Como parte do presente e estudo, foi elaborado um minicurso com o tema: "Números Complexos e Quatérnios: Dos Contextos Históricos ao uso dos Softwares Educativo Livre GeoGebra e QUATERNION, o qual foi apresentado a um grupo de alunos de Licenciatura em Matemática. O conteúdo apresentado, originou-se a partir do projeto de pesquisa "Ensino e aprendizagem de matemática não só como a arte pela arte, mas também, como modelos representativos do mundo real: Saberes e práticas dos professores em formação inicial e continuada" no qual os professores orientadores são colaboradores. 
Para tanto, aborda-se a formulação do conjunto dos Números Complexos, no qual é apresentado seu contexto histórico, definições e propriedades evidenciando a relação entre a rotação no plano bidimensional com os Números Complexos e a relação entre rotação no espaço tridimensional com os Quatérnios. As definições e propriedades foram apresentadas com o auxílio dos Softwares Educativo Livre GeoGebra e Software Livre online QUATERNION, com isso, busca-se facilitar a compreensão e proporcionar uma aprendizagem significativa de conceitos abstratos. Foi aberto um espaço para os participantes fazerem perguntas, e ao final foi aplicado um questionário simples, nos quais, os participantes puderam relatar sobre o uso das Tecnologias Educacionais em especial dos Softwares para o ensino de Matemática.

Utilizando o Software Educativo Livre GeoGebra, durante o minicurso foi resolvido um exercício prático, com o intuito de estimular nos minis cursistas o trabalho com este recurso pedagógico diferenciado (o GeoGebra). A atividade "Dado um Número Complexo qualquer no Plano Complexo (Argand-Gauss ou Diagrama de Argand), construa um quadrado inscrito numa circunferência”, foi respondida passo a passo conforme ilustram as figuras (8 - 16).

Na figura 8, é apresentado a tela inicial do Software GeoGebra; na figura 9 na janela algébrica, escreve-se as coordenadas conforme consolidada por Hamilton; na figura 10, após prosseguir com o comando na janela algébrica o programa escreve os Números Complexos conforme a definição mais usual. Já na figura 11, há a criação de um vetor ligando o ponto de origem com a extremidade de " $(2,3 i)$ ", para representar melhor a visualização da construção pretendida. Logo na figura 12, na janela algébrica é acionado o novo Número Complexo em forma de pares ordenados. Na figura 13, apresenta-se os números imaginários na sua forma usual, e ainda, é criado um novo número complexo " $z_{-} 3$ ”, bem como seus vetores correspondentes para melhor visualização dos participantes, conforme ilustra a figura. Na figura 14, é construído um losango com a ferramenta "polígonos regulares" e, utilizou-se a ferramenta "ângulo" para mostrar a angulação entres os vetores criados anteriormente. Na figura 15, com auxílio da ferramenta "Círculo dados Centro e Um de seus Pontos" é criada uma circunferência e, por fim, na figura 16, é ilustrado a construção da atividade finalizada, sendo realizada uma pequena rotação de modo que o seguimento de reta $\overline{Z_{3} Z_{4}}$, ficasse paralelo ao eixo do Reais. 
Figura 8

Figura 9

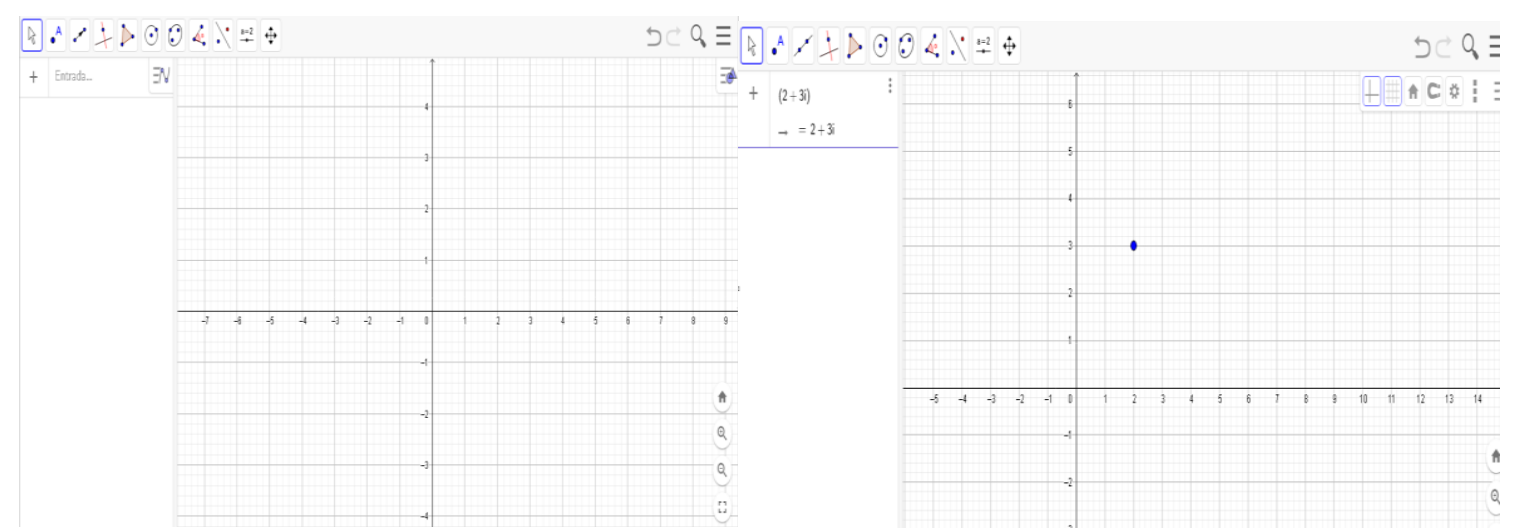

Fonte: Elaborado pelos autores.

Fonte: Elaborado pelos autores.

Figura 10

Figura 11

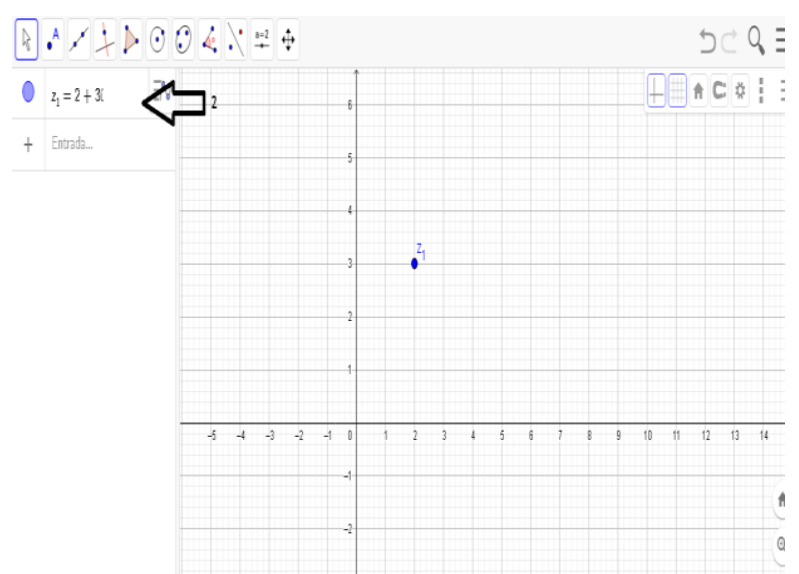

$D C Q \equiv A \cdot A+D \odot \odot<\cdot+4$ $D C Q \equiv$
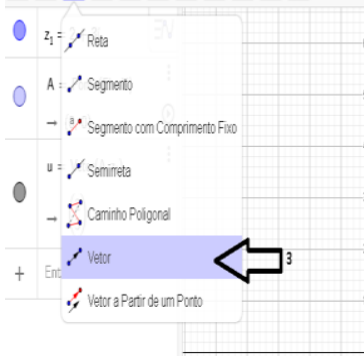

Fonte: Elaborado pelos autores.

Fonte: Elaborado pelos autores.

Figura 13

Figura 12
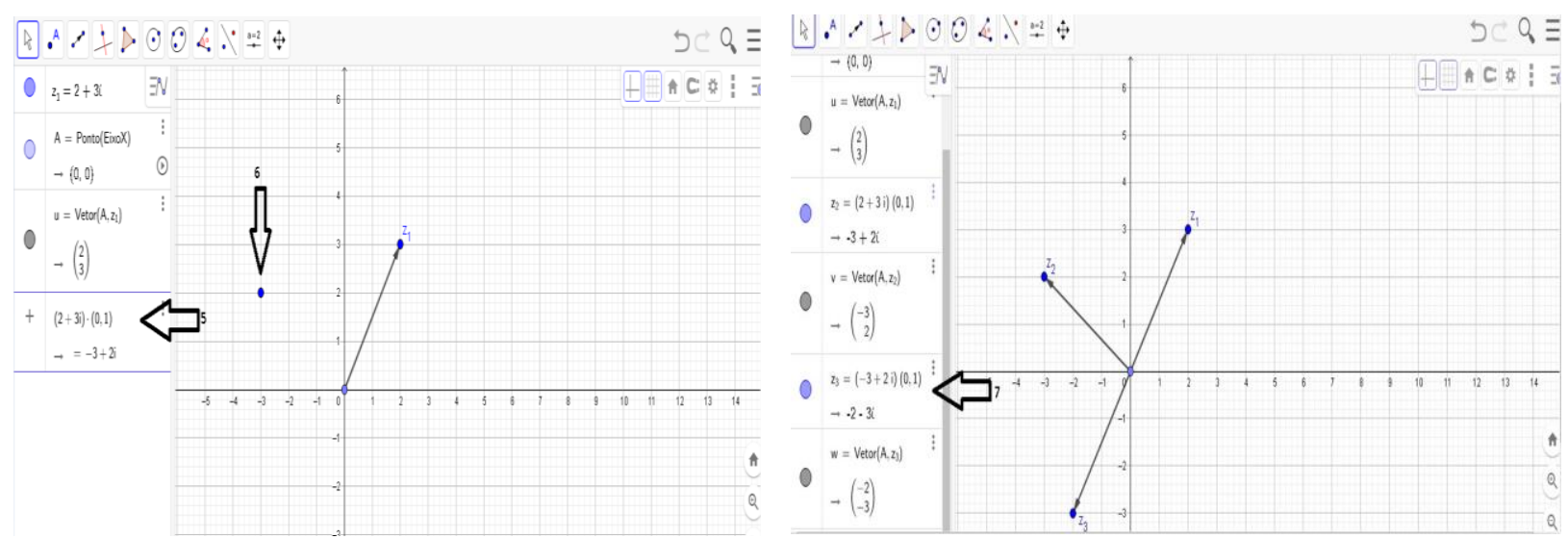

Fonte: Elaborado pelos autores.

Fonte: Elaborado pelos autores. 
Figura 14

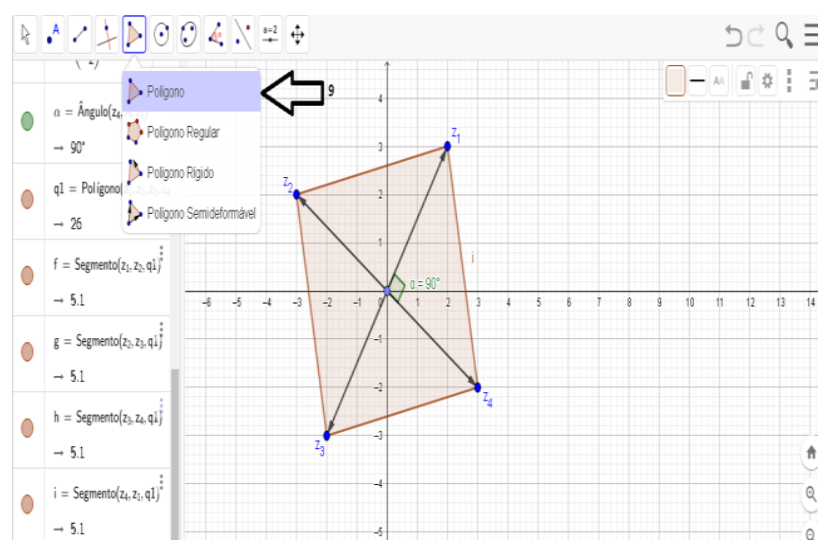

Fonte: Elaborado pelos autores.
Figura 15

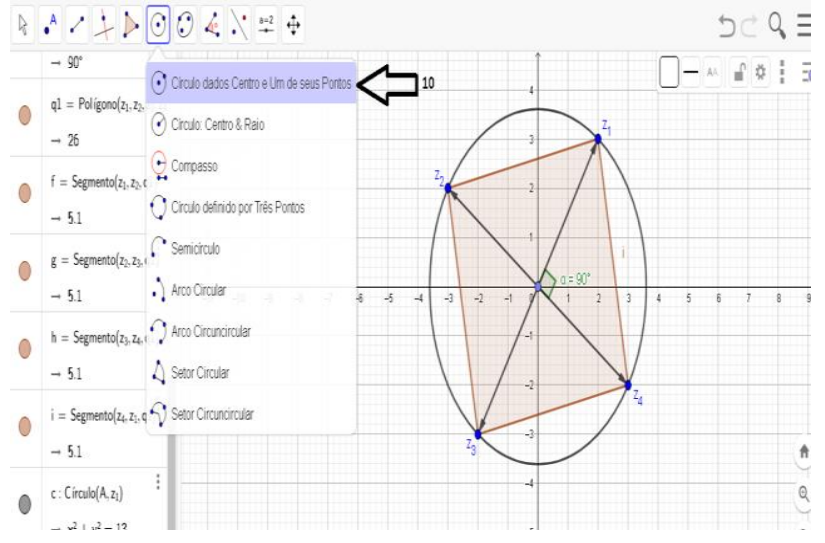

Fonte: Elaborado pelos autores.

Figura 16

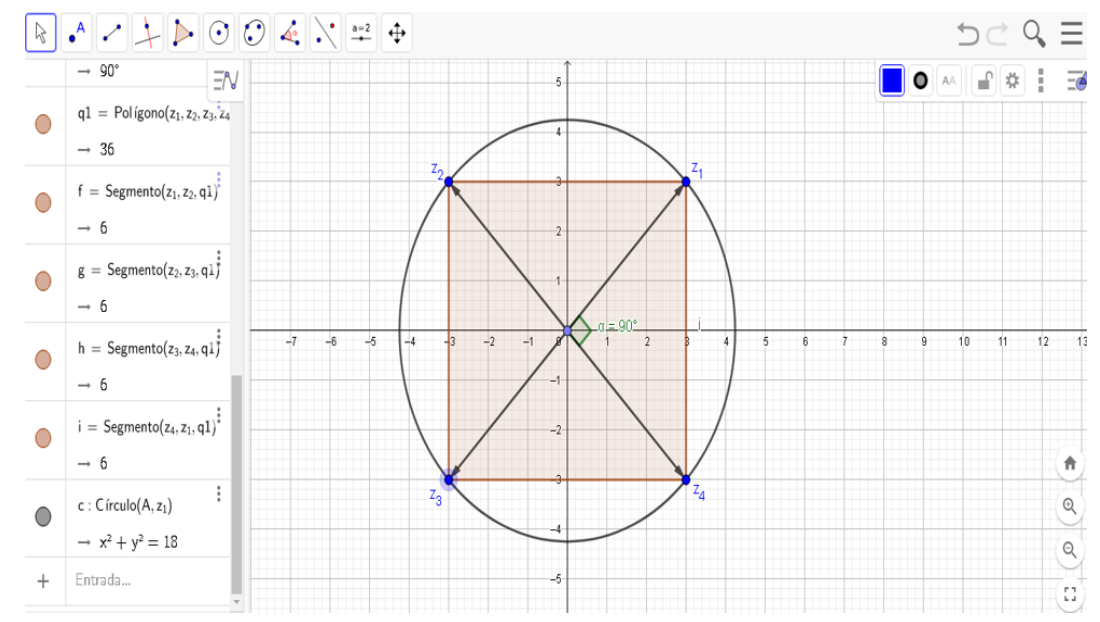

Fonte: Acervo Próprio Autor

Durante o minicurso foi aplicado um questionário aos participantes, que responderam anonimamente; ele foi composto por 6 (seis) perguntas, cujos resultados são apresentados a seguir. Os resultados de duas questões são apresentados graficamente, para as outras 4 (quatro) perguntas são apresentadas algumas das respostas obtidas, identificando os participantes com letras (participante A, participante B, participante C, ...). Para evitar ambiguidades, foram selecionadas as repostas mais completas.

As duas primeiras perguntas estão relacionadas com a identificação dos participantes através da ocupação e da IES a qual estão vinculados. O gráfico 1 apresenta 
o curso e a Instituição de Ensino Superior de cada participante do minicurso, nota-se que houve interesse de alunos de outras instituições e inclusive de outros Estados

Gráfico 1: Distribuição por IES dos participantes do curso

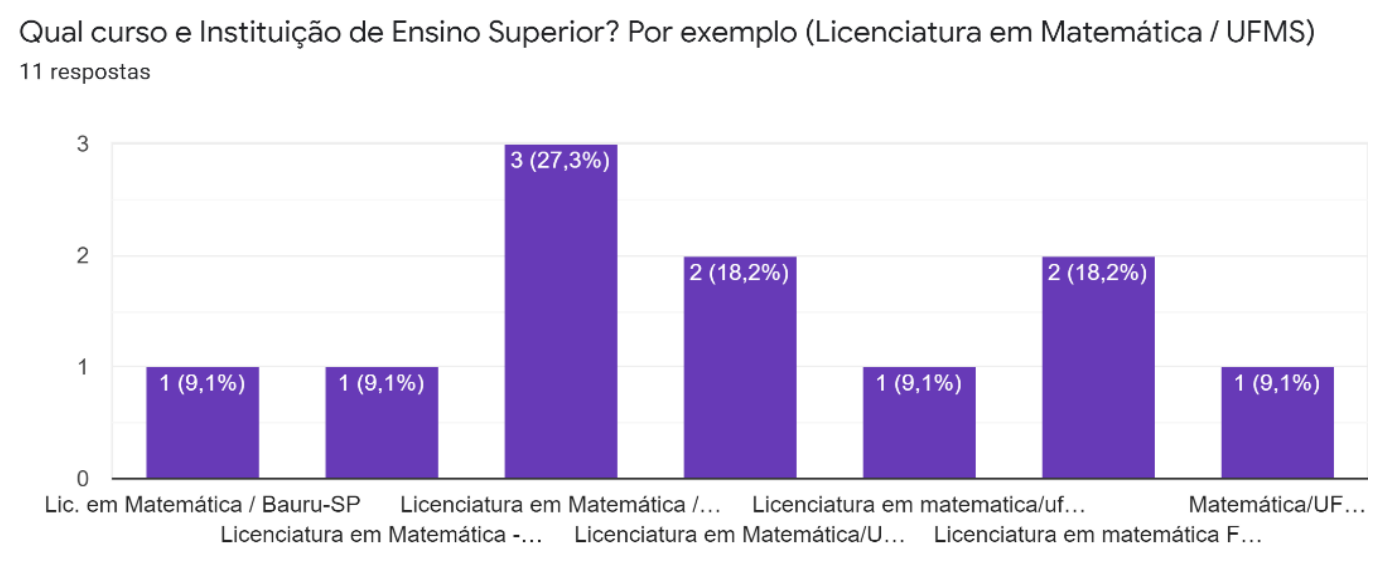

Fonte: Os autores.

- Na segunda pergunta os participantes foram questionados em relação à sua ocupação principal, mostrando que eles eram estudantes ou professores, sendo que a maioria deles $(90,9 \%)$ é estudante.

Gráfico 2: Ocupação dos participantes

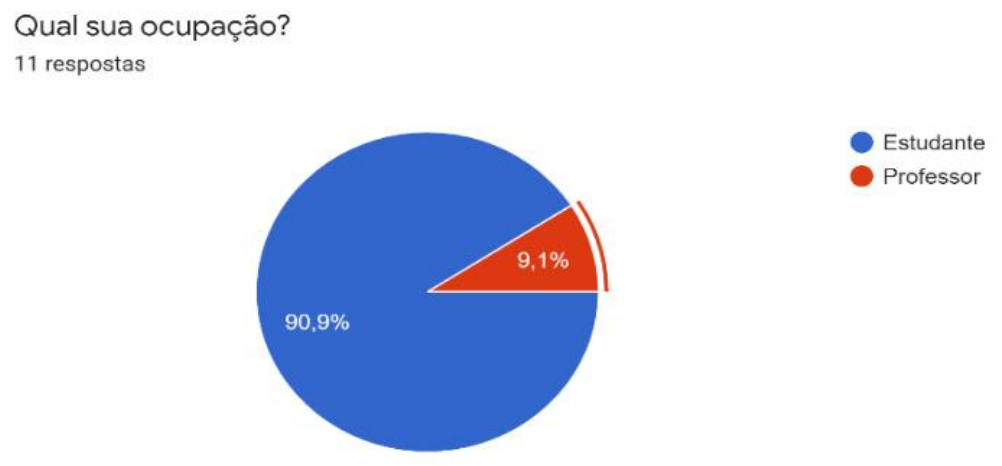

Fonte: Os autores.

Continuando, a terceira pergunta questiona se os participantes já conheciam a classe dos números hiper complexos chamado Quatérnios? Como resposta, o(a) participante F comenta que, "Já tinha conhecimento da classe. Minha iniciação cientifica aborda a classe dos números complexos e dos quatérnios, o que me despertou interesse 
nesta edição da SEMAT". Os demais participantes comentaram que não conheciam, ou conheciam muito pouco.

$\mathrm{Na}$ quarta pergunta foi questionado: "Na sua opinião, o uso dos softwares GeoGebra e Quaternion podem ser utilizados como método alternativo para o ensino de conceitos abstratos, como os Números Complexos e Quatérnios?" O(a) participante A respondeu que: "Sim, acredito que os dois softwares se complementam, enriquecendo as possíveis demonstrações de conceitos abstratos, com as limitações do GeoGebra, 0 QUATERNION se tem mostrado muito importante pra essa área". $\mathrm{O}(\mathrm{a})$ participante $\mathrm{C}$ respondeu que, "Sim, o uso de software contribui muito para o ensino". O(a) participante E respondeu que, "Certamente, a maneira como foi apresentado nesse minicurso mostrou essa possibilidade na prática". $\mathrm{O}(\mathrm{a})$ participante $\mathrm{H}$ respondeu que, "Sim, creio que fica menos abstrato e mais fácil de visualizar". E ainda, os(as) outros(as) componentes disseram que sim os softwares apresentados são facilitadores no ensino e aprendizagem de conteúdos matemáticos abstratos.

No quinto item, foi perguntado: "O contexto apresentado no minicurso a respeito dos números hiper complexos, os Quatérnios, contribuirá com sua formação?” O(a) integrante B responde que, "Todo conhecimento vem a contribuir para nossa formação acadêmica, tanto quanto pessoal". Logo, o(a) integrante D responde que, "Sim, pois são conceitos novos que ainda não tinha visto na graduação". E em consonância com as repostas anteriores os outros mini cursistas apontam que sim, o aprendizado dos Quatérnios contribuirá para suas formações.

No sexto e último item, perguntou-se: "Cite três pontos positivos em utilizar softwares para fins educacionais, como por exemplo para o ensino de matemática nos cursos de Licenciatura em Matemática". Entre todos os pontos positivos relatados pelos(as) praticantes, temos: "softwares tornam as aulas mais divertidas, uma proximidade a mais na área tecnológica, melhores visualizações, cálculos instantâneos, estímulo da criatividade, conceitos algébricos ficam concretos, estimula a investigação, melhora a dinâmica e interação com os alunos, além desses pontos, o(a) participante G relata que, "Creio que o uso desses softwares para fins educativos podem facilitar 0 entendimento dos estudantes sobre um coisa que parece de início, um pouco complexa, já diz o nome do conjunto. Também pode ser muito eficaz em concretizar esses conhecimentos, pois aplicando em imagens, fica um conteúdo muito menos abstrato." 


\section{ENSIN@UFMS 2021}

ISSN 2525-7056

Finalmente, o(a) componente I aponta que: “i) a manipulação do software permite maior manipulação do objeto matemático (funções, geometria e afins) por parte dos alunos; ii) a precisão dos gráficos e a rapidez na sua construção otimiza o tempo da aula permitindo tempo maior para discussões entres alunos e entre alunos e professor; iii) a partir das condições dadas no item ii), é possível analisar o comportamento da função em seus aspectos gráficos, permitindo assim a exploração de conceitos desta função entre coisas a mais".

\section{Considerações Finais}

Este trabalho teve como propósito, apresentar uma abordagem dos Números Complexos constituída por Willian Rowan Hamilton, e sua história no desenvolvimento dos Quatérnios como extensão do Corpo dos Complexos. Foi exposto a estudantes de licenciatura em Matemática um minicurso em que foram apresentados os Números Hiper Complexos. Acredita-se que esta experiência de envolvimento com as tecnologias educacionais permitiu aos participantes vivenciar e perceber a importância de utilizar tais recursos tecnológicos dentro da sala de aula, bem como vivenciar algumas das dificuldades que podem ser encontradas neste percurso. Pelas respostas recebidas, é possível entrever que o desenvolvimento desta atividade Ihes proporcionou oportunidades para constituir conhecimentos referentes ao uso de Softwares no ensino de Matemática nos diversos níveis da educação. Também, é importante levar em consideração que autores como Ferreira (2012), apontam por mudanças tanto na formação inicial quanto continuada dos professores em relação utilização das Tecnologias Educacionais no contexto escolar, bem como, proporcionar o aprimoramento de habilidades relacionadas à aplicação desses conceitos intangíveis na resolução de problemas da realidade dos alunos.

\section{REFERÊNCIAS BIBLIOGRÁFICAS}

AZEVEDO SILVA, M. M; PEREIRA, J. S; SOUSA, E. K. V. de. Números complexos: de gauss às aplicações no geogebra. Boletim Cearense de Educação e História da Matemática, v. 5, n. 14, p. 213-222, 2018.

BRASIL. Conselho Nacional de Educação. Diretrizes Curriculares Nacionais de Matemática, Bacharelado e Licenciatura. Parecer CNE/CES, 2001, 1302. Disponível em: http://portal.mec.gov.br/cne/arquivos/pdf/CES13022.pdf. Acesso em: 03 mai. 2021. 
FERREIRA, T. B. Novas tecnologias educacionais e mediação pedagógica: uma relação possível na universidade. Colóquio internacional "Educação e Contemporaneidade", 6., 2012, São Cristóvão. Anais eletrônicos. São Cristóvão: EDUCON, 2012. Disponível em: https://ri.ufs.br/jspui/handle/riufs/10177. Acesso em: 24 jun. 2021.

GALLENBERGER, D. Software QUATERNION. Verão 1., 2021. Disponível em: https://quaternions.online/. Acesso em: 20 jun. 2021.

GOMES, A. S. et al. Avaliação de software educativo para o ensino de matemática. In: WIE 2002 Workshop Brasileiro de Informática Educativa. Florianópolis: SBC. 2002.

GUEDES JUNIOR, R. R. et al. Números complexos: desenvolvimento e aplicações. 2016.

HOHENWARTER, M. GeoGebra Classic 5 - Software de geometria dinâmica. Versão 5.0.,2016. Disponível em: https://www.geogebra.org/?lan=pt. Acesso em: 20 jun. 2021.

NEVES, R. C. Os quatérnios de Hamilton e o Espaço. UFRJ - Instituto de Matemática Programa de Pós-Graduação em Ensino de Matemática Mestrado em Ensino de Matemática. 2008.

NEVES, R. C. Aplicações de Números Complexos em Geometria. Instituto Nacional de Matemática Pura e Aplicada, Mestrado Profissional em Matemática em Rede Nacional. 2014.

SANTOS, M. A. dos. Dos números complexos aos quatérnions: desenvolvimento algébrico, interpretação geométrica e aplicações. Dissertação de Mestrado. Universidade Tecnológica Federal do Paraná. 2013.

SEMAT, Semana Acadêmica de Matemática, Campus de Três Lagoas, 2021. Universidade Federal de Mato Grosso do Sul. Disponível em: https://sematufmscptl.wixsite.com/meusite. Acesso em: 25 jun. 2021.

SILVA, C. A. da. Modelagem e tecnologia: alternativas metodológicas para a educação matemática. Dissertação (Mestrado). Universidade Estadual Paulista (Unesp), Faculdade de Filosofia e Ciências, Marília. 2019.

SOFFA, M. M; ALCÂNTARA, P. R. de Carvalho. O uso do software educativo: reflexões da prática docente na sala informatizada. In: Congresso Nacional de Educação (EDUCERE). 2008. 


\section{NOTAS}

\section{IDENTIFICAÇÃO DE AUTORIA}

Alessandro Ribeiro da Silva. Bolsista do Grupo PET Conexões de Saberes Matemática. Graduando do Curso de Licenciatura em Matemática da Universidade Federal de Mato Grosso do Sul (UFMS), Campus de Três Lagoas (CPTL), Três Lagoas, MS, Brasil.

E-mail: alessandro.ribeiro@ufms.br

(D) https://orcid.org/0000-0001-8012-2072

Renato César da Silva. Doutor em Engenharia Mecânica pela Escola de Engenharia de São Carlos da Universidade de São Paulo (USP). Professor da Universidade Federal de Mato Grosso do Sul (UFMS), Campus de Três Lagoas (CPTL), Três Lagoas, MS, Brasil.

E-mail: renato.silva@ufms.br

(1) https://orcid.org/0000-0002-7931-7785

Edivaldo Romanini. Doutor em Engenharia Mecânica pela Universidade Estadual de Campinas (UNICAMP). Professor da Universidade Federal de Mato Grosso do Sul (UFMS), Campus de Três Lagoas (CPTL), Três Lagoas, MS, Brasil.

E-mail: edivaldo.romanini@ufms.br

느 https://orcid.org/0000-0001-5382-0326

\section{AGRADECIMENTOS}

Os autores agradecem à Universidade Federal de Mato Grosso do Sul e ao Programa de Educação Tutorial.

\section{FINANCIAMENTO}

Bolsa do Programa de Educação Tutorial.

\section{CONSENTIMENTO DE USO DE IMAGEM}

Não se aplica.

\section{APROVAÇÃO DE COMITÊ DE ÉTICA EM PESQUISA}

Não se aplica.

\section{LICENÇA DE USO}

Autores mantêm os direitos autorais e concedem à revista ENSIN@ UFMS - ISSN 2525-7056 o direito de primeira publicação, com o trabalho simultaneamente licenciado sob a Licença Creative Commons Attribution (CC BY-NC-SA 4.0), que permite compartilhar e adaptar o trabalho, para fins não comerciais, reconhecendo a autoria do texto e publicação inicial neste periódico, desde que adotem a mesma licença, compartilhar igual.

\section{EDITORES}

Patricia Helena Mirandola Garcia, Eugenia Brunilda Opazo Uribe, Gerson dos Santos Farias.

\section{HISTÓRICO}

Recebido em: 21/07/2021 - Aprovado em: 30/09/2021 - Publicado em: 10/12/2021.

\section{COMO CITAR}

SILVA, A. R; SILVA, R. C; ROMANINI, E. Números Complexos e Quatérnios: Dos Contextos Históricos ao uso do Software Educativo Livre GeoGebra e Software Livre on-line Quaternion. Revista ENSIN@ UFMS, Três Lagoas, v. 2, n. 6, p. 266-290. 2021. 